\title{
EMPEZAR CON BUEN PIE: INSERCIÓN A LA ENSEÑANZA PARA PROFESORES PRINCIPIANTES
}

\section{STARTING OFF RIGHT: TEACHING INSERTION OF BEGINNING TEACHERS}

Carlos Marcelo*

Resumen: Los profesores, en su proceso de aprendizaje, pasan por diferentes etapas. De todas estas fases la que más nos interesa en este artículo es la que se inicia con los primeros contactos con la realidad de la escuela, asumiendo el papel profesional reservado a los docentes. La inserción profesional en la enseñanza es el periodo de tiempo que abarca los primeros años, en los cuales los profesores han de realizar la transición desde estudiantes a docentes. Es un periodo de tensiones y aprendizajes intensivos en contextos generalmente desconocidos y durante el cual los profesores principiantes deben adquirir conocimiento profesional además de conseguir mantener un cierto equilibrio personal. Los programas de inserción profesional para los profesores principiantes son una verdadera alternativa para favorecer que los primeros años como docentes no sean años frustrantes, a lo que hemos denominado "aterriza como puedas”.

Palabras claves: Profesorado principiante. Programas de inserción. Mentor. Desarrollo profesional.

Abstract: Teachers go throughout different stages during the processes of learning to teach. This article is concerned with the stage that starts with the first contacts with the school reality, in which teachers embrace their teaching roles. Professional insertion in teaching includes the first years of teaching in which teachers make the transition from students to teachers. It is a period of tension, and intensive learning in contexts usually unknown, and it is during this period that beginning teachers have to acquire professional knowledge, and maintain a certain personal balance. The programs of professional insertion for beginning teachers are good alternatives to support the first years of teaching so that they do not become years of frustration, or what have been called "do your best year”.

Keywords: Beginning teachers. Insertion Programs. Mentor. Professional Development.

\section{Introducción}

Vivimos tiempos de cambio. Cambios sociológicos, económicos, valóricos, demográficos, culturales, etc. que están desafiando constantemente la capacidad de equilibrio, integración e innovación de nuestras sociedades y sistemas. Una de las características de la sociedad en la que vivimos tiene que ver con el hecho de que el conocimiento es uno de los principales valores de sus ciudadanos. El valor de las sociedades actuales está directamente relacionado con el nivel de formación de sus ciudadanos, y de la capacidad de innovación y emprendimiento que estos posean. Pero los conocimientos,

\footnotetext{
* Catedrático de Didáctica de la Universidad de Sevilla. España. Director del Grupo de Investigación IDEA: http:// prometeo.us.es/idea. E-mail: <marcelo@us.es>.

* Professor of Didactics at the University of Sevilla. Spain. Director of the Research Group IDEA: http://prometeo. us.es/idea. E-mail: <marcelo@us.es>.
} 
en nuestros días, tienen fecha de caducidad y ello nos obliga ahora más que nunca a establecer garantías formales e informales para que los ciudadanos y profesionales actualicen constantemente su competencia. Hemos entrado en una sociedad que exige de los profesionales una permanente actividad de formación y aprendizaje.

¿En qué afectan estos cambios a las escuelas y al trabajo que en ellas desarrolla el profesorado? ¿Cómo debemos repensar el trabajo del docente en estas nuevas circunstancias? ¿Cómo deberían formarse los nuevos profesores? ¿Qué características debería de tener la profesión docente en el siglo XXI para ser realmente una profesión del conocimiento? (MARCELO, 2002).

\section{El proceso de convertirse en profesor: un continuo de aprendizaje a lo largo de la vida}

Convertirse en profesor es un largo proceso. A las instituciones de formación inicial del profesorado llegan candidatos que no son "vasos vacíos". Como ya investigara Lortie (1975), las miles de horas de observación como estudiantes contribuyen a configurar un sistema de creencias hacia la enseñanza que los aspirantes a profesores tienen y que les ayudan a interpretar sus experiencias en la formación. Estas creencias a veces están tan arraigadas que la formación inicial no consigue el más mínimo cambio profundo en ellas (PAJARES, 1992; RICHARDSON; PLACIER, 2001).

La formación inicial del profesorado ha sido objetivo de múltiples estudios e investigaciones (COCHRAN-SMITH; FRIES, 2005). En general se observa una gran insatisfacción tanto de las instancias políticas como del profesorado en ejercicio o de los propios formadores respecto a la capacidad de las actuales instituciones de formación para darrespuesta a las necesidades actuales de la profesión docente. Las críticas hacia su organización burocratizada, el divorcio entre la teoría y la práctica, la excesiva fragmentación del conocimiento que se enseña, la escasa vinculación con las escuelas (FEIMAN-NEMSER, 2001) están haciendo que ciertas voces críticas propongan reducir la extensión de la formación inicial para incrementar la atención al periodo de inserción del profesorado en la enseñanza. Es el caso del reciente informe de la OCDE - Organización para la Cooperación y el Desarrollo Económico.

Frente a estas propuestas, viene bien recordar el excelente artículo escrito por David Berliner (2000) en el que refuta una docena de críticas que habitualmente se hacen a la formación inicial del profesorado (que para enseñar basta con saber la materia, que enseñar es fácil, que los formadores de profesores viven en una torre de marfil, que los cursos de metodología y didáctica son asignaturas blandas, que en la enseñanza no hay principios generales válidos, etc). Críticas, desde el punto de vista del autor, interesadas y con una visión bastante estrecha de la contribución que la formación inicial tiene en la calidad del profesorado. Dice Berliner (2000, p. 370): “creo que se ha prestado poca atención al desarrollo de aspectos evolutivos del proceso de aprender a enseñar, desde la formación inicial, la inserción a la formación continua”. En este proceso, la formación inicial juega un papel importante y no baladí o sustituible como algunos grupos o instituciones están sugiriendo.

Los profesores, en su proceso de aprendizaje, pasan por diferentes etapas. De todas estas fases la que más nos interesa en este informe es la que se inicia con los primeros contactos con la realidad de la 
escuela, asumiendo el papel profesional reservado a los docentes. Bransford, Darling-Hammond y LePage (2005) han planteado que, para dar respuesta a las nuevas y complejas situaciones con las que se encuentran los docentes, es conveniente pensar en los profesores como expertos adaptativos, es decir, personas preparadas para un aprendizaje eficiente a lo largo de toda la vida. Esto es así porque las condiciones de la sociedad son cambiantes y cada más se requiere personas que sepan combinar la competencia con la capacidad de innovación.

Los profesores principiantes necesitan poseer un conjunto de ideas y habilidades críticas así como la capacidad de reflexionar, evaluar y aprender sobre su enseñanza de tal forma que mejoren continuamente como docentes. Ello es más posible si el conocimiento esencial para los profesores principiantes se pudiera organizar, representar y comunicar de forma que les permita a los alumnos una comprensión más profunda del contenido que aprenden.

\section{Los problemas de los profesores principiantes}

La inserción profesional en la enseñanza, es el periodo de tiempo que abarca los primeros años, en los cuales los profesores han de realizar la transición desde estudiantes a profesores. Es un periodo de tensiones y aprendizajes intensivos en contextos generalmente desconocidos $\mathrm{y}$ durante el cual los profesores principiantes deben adquirir conocimiento profesional además de conseguir mantener un cierto equilibrio personal. Es éste el concepto de inserción que asume Vonk, autor holandés con una década de investigaciones centradas en éste ámbito: "definimos la inserción como la transición desde profesor en formación hasta llegar a ser un profesional autónomo. La inserción se puede entender mejor como una parte de un continuo en el proceso de desarrollo profesional de los profesores" (VONK, 1996, p. 115).

Conviene insistir en esta idea de que el periodo de inserción es un periodo diferenciado en el camino de convertirse en profesor. No es un salto en el vacío entre la formación inicial y la formación continua sino que tiene un carácter distintivo y determinante para conseguir un desarrollo profesional coherente y evolutivo (BRITTON et al. 2003). El periodo de inserción y las actividades propias que le acompañan varían mucho entre los países. En algunos casos se reducen a actividades burocráticas y formales. En otros casos, como veremos más adelante, configuran toda una propuesta de programa de formación cuya intención es asegurar que los profesores entren en la enseñanza acompañados por otros que pueden ayudarle.

Los profesores principiantes tienen, según Feiman-Nemser (2001), dos tareas que cumplir: deben enseñar y deben aprender a enseñar. Independientemente de la calidad del programa de formación inicial que hayan cursado, hay algunas cosas que sólo se aprenden en la práctica y ello repercute en que este primer año, sea un año de supervivencia, descubrimiento, adaptación, aprendizaje y transición. Las principales tareas con que se enfrentan los profesores principiantes son: adquirir conocimientos sobre los estudiantes, el currículo y el contexto escolar; diseñar adecuadamente el currículo y la enseñanza; comenzar a desarrollar un repertorio docente que les permita sobrevivir como profesor; crear una comunidad de aprendizaje en el aula, y continuar desarrollando una identidad profesional. Y el problema es que esto deben hacerlo en general cargados con las mismas 
responsabilidades que los profesores más experimentados (MARCELO, 1999).

Ya resulta clásico el trabajo desarrollado por Simon Veenman (1984) quien popularizó el concepto de "choque con la realidad" para referirse a la situación por la que atraviesan muchos docentes en su primer año de docencia. Según este autor holandés, el primer año se caracteriza por ser, en general, un proceso de intenso aprendizaje -del tipo ensayo-error en la mayoría de los casos-, y caracterizado por un principio de supervivencia, y por un predominio de valor de lo práctico. Los programas de iniciación tratan de establecer estrategias para reducir o reconducir el denominado "choque con la realidad". Los profesores principiantes se encuentran con ciertos problemas específicos de su estatus profesional. Valli (1992) plantea que son la imitación acrítica de conductas observadas en otros profesores; el aislamiento de sus compañeros; la dificultad para transferir el conocimiento adquirido en su etapa de formación y el desarrollo de una concepción técnica de la enseñanza, los problemas que más amenazan a los profesores principiantes. En una revisión más reciente, Britton et al (2003) y Serpell (2000) confirman que los problemas encontrados por Veenman siguen siendo actuales: cómo gestionar el aula, cómo motivar a los alumnos, cómo relacionarse con los padres y con los compañeros, en definitiva, cómo sobrevivir personal y profesionalmente.

Los primeros años de docencia no sólo representan un momento de aprendizaje del "oficio" de la enseñanza, especialmente en contacto con los alumnos en las clases. Significan también un momento de socialización profesional. Es durante las prácticas de enseñanza que los futuros profesores empiezan a conocer la "cultura escolar" (KENNEDY, 1999). Pero es durante el periodo de inserción profesional cuando esta socialización se produce con mayor intensidad. En este momento, los nuevos profesores aprenden e interiorizan las normas, valores, conductas, etc., que caracterizan a la cultura escolar en la que se integran.

\section{Los profesores principiantes y la cultura profesional}

El periodo de iniciación a la enseñanza representa el ritual que ha de permitir transmitir la cultura docente al profesor principiante (los conocimientos, modelos, valores y símbolos de la profesión), la integración de la cultura en la personalidad del propio profesor, así como la adaptación de éste al entorno social en que lleva a cabo su actividad docente. Dicha adaptación puede ser fácil cuando el entorno sociocultural coincide con las características del profesor principiante. Sin embargo, tal proceso puede ser más difícil cuando debe integrarse a culturas que le son desconocidas hasta el momento de empezar a enseñar. Sabar (2004) hace el símil entre los procesos de socialización del profesor principiante $\mathrm{y}$ de los inmigrantes. Al igual que los inmigrantes se desplazan a un país cuya lengua normalmente no conocen, así como su cultura y normas de funcionamiento, así

extraño que a menudo no está familiarizado con las normas y símbolos aceptados en la escuela o con los códigos internos que existen entre profesores y alumnos. En este sentido los profesores principiantes parecen recordar a los inmigrantes que abandonan una cultura familiar para moverse a un lugar atractivo y a la vez repelente. (COLLIS; WINNIPS, 2002).

Otros investigadores que han revisado el periodo de inserción, así como los 
programas que se han puesto en marcha, han sido Wideen, Mayer-Smith y Moon (1998). Después de su revisión, concluyen que ha habido mucha investigación sobre el primer año de enseñanza, confirmándose la visión ampliamente extendida de que este año supone un choque cultural para los profesores principiantes, especialmente para los que están peor preparados. Un aspecto negativo que señalan los autores es el siguiente:

encontramos una población homogénea de profesores principiantes intentando aprender a enseñar a una población heterogénea de alumnos en las escuelas. También encontramos muchos programas que tienen muy poco efecto sobre las creencias fuertemente asentadas acerca de la enseñanza que los profesores traen a sus programas de formación. Tales programas frecuentemente parecen tener propósitos cruzados con las experiencias que los profesores en formación se encuentran durante sus prácticas de enseñanza y su primer año como docentes. (WIDEEN; MAYER-SMITH; MOON, 1998, p. 159).

Estos programas sólo cumplen una función burocrática pero no contribuyen a crear una identidad profesional en los docentes.

En este proceso de inserción en una nueva cultura (en muchos casos no tan nueva ya que algunos aspectos resultan reconocibles debido a las miles de horas de aprendizaje por observación como alumno), los profesores principiantes abandonan conocimientos adquiridos en su formación inicial. En un informe sobre la formación del profesorado en Europa, Buchberger, Campos, Kallos y Stephenson (1997, p. 54) concluyen que muchos aspectos positivos de la formación inicial del profesorado se pierden cuando los profesores principiantes llegan a las escuelas. Afirman que

\begin{abstract}
Aunque esta pérdida de competencia significa una pérdida de recursos individuales y públicos, no ha llevado aún a un esfuerzo sistemático en la mayor parte de los estados miembros de la Unión Europea. Además, la mayoría de las escuelas en toda Europa aún no han desarrollado una "cultura de la inserción" para los profesores principiantes.
\end{abstract}

\section{Mejorar la retención y la calidad docente a través de los programas de inserción profesional}

Como hemos visto, los primeros años de docencia son fundamentales para asegurar un profesorado motivado, implicado y comprometido con su profesión. Tradicionalmente el periodo de inserción profesional en la docencia se ha considerado según el modelo "nada o húndete" o como yo mismo lo denominaba en otro trabajo "aterriza como puedas" (MARCELO, 1999). En realidad, si observamos cómo las profesiones incorporan y socializan a los nuevos miembros, nos daremos cuenta del grado de desarrollo y estructuración que estas profesiones tienen. No es común que un médico recién egresado deba de realizar una operación de trasplante de corazón. Ni mucho menos que a un arquitecto con poca experiencia se le asigne la construcción de un edificio de viviendas. No digamos de un piloto con pocas horas de vuelo que se le deje comandar un Aerobús 340. Podríamos poner más ejemplos que nos mostrarían que las profesiones intentan proteger su propio prestigio y la confianza de la sociedad y de sus clientes asegurándose que los nuevos miembros de la profesión tienen las competencias apropiadas para ejercer el oficio. Algo así ocurría en la Edad Media con los gremios. 
¿Qué podríamos pensar de una profesión que deja para los nuevos miembros las situaciones más conflictivas y difíciles? Algo así ocurre en la enseñanza. En general se ha venido reservando a los profesores principiantes los centros educativos más complejos y las aulas y horarios que los profesores con más experiencia han desechado. Pero esta realidad empieza a cambiar sobre todo motivada por las causas que anteriormente hemos expuesto y que tienen que ver con el diagnóstico hecho por los informes internacionales que muestran que o cuidamos los primeros años de enseñanza o tendremos que replantear la función de la escuela en nuestra sociedad. El informe de la OCDE al que me he referido a lo largo de este trabajo lo deja claro cuando afirma:

Incluso en los países que no tienen problemas para incorporar a profesores, la falta de atención hacia los profesores principiantes tiene un costo a largo plazo. La calidad de la experiencia profesional en los primeros años de docencia se entiende en estos momentos como de una influencia determinante en la probabilidad de abandonar la profesión docente. Los programas de inserción y apoyo a los profesores principiantes pueden mejorar los porcentajes de retención de profesores, mejorando la eficacia y la satisfacción de los profesores principiantes con la enseñanza. (OCDE, 2005, p. 117).

En un libro titulado Comprehensive Teacher Induction, Britton, Paine, Pimm y Raizen (2003) realizaron una revisión de programas de formación de profesores principiantes en diferentes países a los que nos referiremos más adelante. Pero han aportado la idea de que los programas pueden ser buenos o no dependiendo no sólo de las actividades que incluyan, sino de los compromisos públicos que asuman, de las metas que se planteen, así como de los esfuerzos y dinámicas que pongan en marcha.

Hay que aclarar que estos programas han de entenderse como una propuesta específica para una etapa que es distinta tanto de la formación inicial como a la de formación en servicio. Los estudios muestran que hay una gran variedad en cuanto a características y contenidos. La duración y la intensidad son dos aspectos importantes. Los programas de inserción pueden variar desde una simple reunión a principio de curso a programas muy estructurados que implican múltiples actividades. Unos están diseñados para hacer crecer a los profesores principiantes mientras que otros están orientados hacia la evaluación y remediar fallas (SMITH; INGERSOLL, 2004).

\section{¿Bajo qué condiciones tienen éxito los programas de inserción?}

Ingvarson, Meiers y Beavis (2005) se plantearon la pregunta: ¿qué componentes de la formación tienen un mayor impacto en la mejora de la enseñanza del profesorado? Para responder a esta pregunta indagaron en cuatro grandes programas de formación continua de profesores en Australia. Reproducimos los hallazgos de esta investigación porque nos ofrecen una considerable luz para responder a la pregunta de este epígrafe: Se trata de programas que:

- Ofrecen a los profesores oportunidades para centrarse en el contenido que los alumnos deben de aprender y en cómo enfrentarse a las dificultades que los alumnos se encontrarán al aprender el contenido;

- Utilizan el conocimiento generado por la investigación acerca del aprendizaje del contenido por los alumnos; 
- Incluyen oportunidades para que los profesores de forma colaborativa puedan analizar el trabajo de los alumnos;

- Buscan que los profesores reflexionen activamente acerca de sus prácticas y las comparen con estándares adecuados de práctica profesional;

- Implican a los profesores para que identifiquen lo que necesitan aprender y planifiquen experiencias de aprendizaje que les permitan cubrir esas necesidades;

- Proporcionan tiempo a los profesores para probar nuevos métodos de enseñanza, $\mathrm{y}$ recibir apoyo $\mathrm{y}$ asesoramiento en sus clases cuando se encuentran con problemas de implementación;

- Incluyen actividades que animan a los profesores a hacer menos privadas sus prácticas de forma que puedan recibir retroacción de sus compañeros. (INGVARSON; MEIERS; BEAVIS; 2005, p. 15-16).

Smith e Ingersoll han llevado a cabo algunos estudios para analizar el efecto que los programas de inserción están teniendo en la reducción del abandono y de la rotación del profesorado principiante. Comentan estos autores que

Un número de estudios parece apoyar la hipótesis de que programas de inserción bien concebidos y bien implementados tienen éxito en mejorar la satisfacción en el trabajo, la eficacia y la retención de los nuevos profesores. (SMITH; INGERSOLL, 2004, p. 684).

Además, encuentran que los profesores que empezaron su carrera como profesores a tiempo completo dejaron la enseñanza en menor proporción que los profesores que la iniciaron como profesores a tiempo parcial. Por otra parte, los profesores de educación especial tenían más probabilidades de dejar la docencia que los demás profesores.

Encontraron tres tipos de programas de inserción:

Inserción básica: que incluye dos componentes: apoyo con el mentor de la misma materia a la que enseña el profesor principiante o de otras diferentes, y la comunicación con el director y/o el jefe del departamento. Estos programas se ofrecen para el 56\% de los profesores principiantes.

Inducción básica + colaboración: incluye el apoyo de cuatro componentes: los profesores tienen el apoyo de un mentor de su misma área de conocimiento, disponen de comunicación con el director o con el jefe del departamento, tienen tiempo para planificar en común con otros colegas y participan en seminarios con otros profesores principiantes. Representan el 26\% de los programas.

Inserción básica + colaboración+red de profesores+recursos extras: Estos programas son los más minoritarios (sólo un 1\%) e incluyen lo anterior pero también participar en una red externa de profesor y una reducción de la carga docente.

Como conclusión de su trabajo, encuentran que algunas actividades parecen ser más eficaces que otras en reducir la rotación de profesorado. "El factor más sobresaliente fue disponer de un mentor de su misma especialidad, tener tiempo para planificar en común con otros profesores de la misma materia y formar parte de una red externa” (SMITH; INGERSOLL, 2004, p. 706).

Por otra parte, hemos de hacer referencia al trabajo de Arends y RigazioDiGilio (2000) en el que revisan los resultados de investigaciones y nos ofrecen la siguiente síntesis: 
- La investigación sobre las preocupaciones de los profesores principiantes indica que los programas de inserción deberían dirigirse a abordar la gestión de clase, la enseñanza, el estrés y carga de trabajo, gestión del tiempo, relaciones con los alumnos, padres, colegas y directivos;

- La calidad de un mentor eficaz tiene que ver con su habilidad para proporcionar apoyo emocional, enseñar sobre el currículo y proporcionar información interna acerca de las normas y procedimientos en la escuela;

- La formación de los mentores repercuten en una mayor eficacia en su trabajo como mentores;

- El contenido de los programas de formación de mentores debería de incluir temas relacionados con el desarrollo y aprendizaje adulto, destrezas de supervisión, habilidades de relación y comunicación;

- Es fundamental la reducción de tiempo o de carga docente para mentores y principiantes;

- Una regulación formal de las reuniones entre profesores principiantes y mentores se relaciona con un éxito en el programa;

- Las tareas de los profesores principiantes deberían de ser más fáciles que las de los profesores con más experiencia;

- Los profesores principiantes valoran y se benefician de la discusión con compañeros principiantes, con profesores de la escuela y con profesores de la universidad;

- Una implicación y apoyo activo del director resulta fundamental;

- Los programas de inserción mejoran la eficacia como docentes de los profesores principiantes y generan satisfacción en ellos;
- No hay evidencias de que los programas de inserción mejoren la proporción de retención a largo plazo de los profesores principiantes.

Aunque algunos hallazgos se repiten respecto a las investigaciones anteriormente revisadas, nos parece que en la síntesis de Wong hay elementos interesantes que muestran las características de los programas de inserción eficientes:

- Metas claramente articuladas;

- Recursos financieros para apoyar las actividades de inserción;

- Apoyo del director de la escuela;

- Mentorazgo a través de mentores experimentados;

- Formación de profesores mentores;

- Reducción de tiempo o de carga docente para profesores principiantes y mentores;

- Reuniones regulares y sistematizadas entre los profesores principiantes y sus mentores;

- Tiempo para que los profesores principiantes observen a los profesores más experimentados;

- Promover una constante interacción entre profesores principiantes y experimentados;

- Talleres para los profesores principiantes antes y a lo largo del año;

- Orientación que incluye cursos sobre temas de interés;

- Duración del programa al menos de uno o dos años;

- Menor énfasis en evaluación y más en asesoramiento y apoyo. (WONG, 2004). 
Estos resultados que hemos revisado nos muestran que existe ya un considerable conocimiento acumulado en relación con los programas de inserción y las características de sus componentes. Vamos a profundizar en ello a continuación.

\section{¿Cuáles son los componentes de los programas de inserción?}

Como podemos comprobar, los programas de inserción ayudan a los profesores a insertarse en la realidad escolar de una forma más adecuada y controlada. Estamos hablando de programas de inserción y quizás convendría detenernos a definir qué entendemos por tal proceso. Zeichner (1979) definió la inserción como

un programa planificado que pretende proporcionar algún tipo de apoyo sistemático y sostenido específicamente a los profesores principiantes durante al menos un año escolar. (ZEICHNER, 1979, p. 6).

Él completa posteriormente su definición diciendo que se refiere a los profesores que han completado su formación inicial, que han recibido su certificación y que han accedido a enseñar en una escuela. Esta definición que hace Zeichner no es comúnmente aceptada porque para muchos la inserción es también el año de prueba que se exige a los profesores principiantes antes de obtener la certificación.

La amplia difusión de programas de inserción y de mentores está creando la necesidad de comprender qué actividades de mentorazgo tienen algún tipo de influencia en los nuevos profesores y en sus alumnos. Los estudios muestran que los beneficios de los programas de inserción y mentorazgo son posibles pero no automáticos. En otro estudio, Ingersoll y Smith (2003) encontraron que participar en un programa de inserción y trabajar con un mentor reduce la probabilidad de que el nuevo profesor deje la enseñanza o se vaya a otra escuela. Pero la mera presencia del mentor no es suficiente. Los mentores deben conocer y tener destreza en sus funciones como mentor.

Hay una visión estrecha y limitada del mentorazgo que lo entiende como una tarea para facilitar la entrada en la enseñanza a los nuevos profesores, ayudándoles en las preguntas e incertidumbres más inmediatas. Y hay una visión más robusta del mentorazgo que lo entiende desde un punto de vista evolutivo en el proceso de aprender a enseñar. Son múltiples, como hemos visto, los componentes de los programas de inserción. Horn, Sterling y Subhan (2002) los agrupan en nueve elementos comunes a los programas de inserción:

1. Orientación: esta actividad es introductoria y se lleva a cabo antes de que empiece el curso para que los nuevos profesores se sitúen en la escuela, el currículo, la comunidad;

2. Mentor: Éste es quizás el factor más importante en los programas de inserción. Muy difundido debido a su bajo coste;

3. Ajustar las condiciones de trabajo. Generalmente se reduce el número de alumnos en las clases de los profesores principiantes, se reducen las actividades extracurriculares, se les proporcionan materiales y recursos, se les proporcionan actividades de formación;

4. Reducción de tiempo para permitir que los profesores principiantes puedan realizar actividades de formación;

5. Desarrollo profesional. Se realizan actividades de formación que pueden 
tener que ver con la enseñanza, gestión de clase y disciplina, relaciones con los padres;

6. Colaboración con compañeros. Esta colaboración es importante porque reduce la sensación de aislamiento. La colaboración puede ser con grupos de profesores que planifican o analizan la enseñanza;

7. Valoración del profesor. Cada cierto tiempo, los profesores principiantes son observados cuando enseñan para detectar sus fortalezas y debilidades.

La idea de que los profesores principiantes requieren un sistema estructurado para apoyar su entrada en la profesión ha ido cambiando en los tiempos recientes, desde considerarse como algo accesorio a entenderse como un elemento central en el proceso de retención del profesorado principiante y de mejora de la calidad de su enseñanza. Ahora resulta generalmente aceptado como un componente fundamental de un enfoque comprehensivo del desarrollo de profesorado.

\section{Para empezar con buen pie}

Por lo revisado a lo largo de este informe, una idea puede quedar clara: ya no podemos seguir ignorando que existe una fase claramente diferenciada en el proceso de convertirse en un buen profesor, que tiene sus propias características y necesidades y que funciona como un eslabón (débil hasta ahora) entre la formación inicial del profesorado y toda su futuro desarrollo profesional. La forma como se aborde el periodo de inserción tiene una importancia trascendental en el proceso de convertirse en un profesor, ya sea en un principiante frustrado o por el contrario en un profesor adaptativo.
Muchos países han venido ignorando la existencia de este periodo formativo. Pero han empezado a preocuparse por él en el momento en el que han percibido la creciente dificultad por incorporar y mantener en la docencia a los mejores profesores, dificultad mayor en determinadas materias del currículo y en las escuelas más desfavorecidas. Si queremos asegurar el derecho de nuestros alumnos a aprender y si queremos que nuestras escuelas sigan siendo espacios donde se construye el conocimiento de las nuevas generaciones, es preciso prestar mucha mayor atención a la forma cómo los nuevos profesores se insertan en la cultura escolar.

Pero hacerlo de forma mecánica puede llevarnos a reproducir modelos de enseñanza que si bien tradicionales, no son los que actualmente vienen demandando la sociedad del conocimiento. El proceso de inserción no debe sólo integrar al nuevo profesorado en la cultura escolar vigente. De ser así, lo único que estaríamos consiguiendo es cerrar el círculo de la socialización perfecta: alumnos que a lo largo de miles de horas han observado a profesores enseñando, vuelven a la escuela a enseñar cómo les enseñaron sus viejos profesores. Para romper ese círculo, para nada virtuoso, necesitamos que el periodo de inserción sea un periodo de indagación, crecimiento, innovación y reflexión. Y para ello resulta fundamental la necesaria participación de las personas e instituciones que pueden ayudar a tener una mirada más compleja hacia la realidad de la escuela y del aula.

Un elemento crucial de los actuales programas de inserción son los profesores mentores, tutores, orientadores, etc. La mayoría de programas de inserción que hemos revisado incluyen la participación de un profesor mentor como vínculo entre lo nuevo y lo antiguo, entre la experiencia 
y la inexperiencia. Sin embargo, existen muchas dudas respecto de las posibilidades de transmitir el conocimiento que fundamentalmente es tácito. En la revisión que realizó, Feiman-Nemser (2001) llamaba la atención acerca de que el entusiasmo por la figura del mentor no se ha visto acompañado con una aclaración acerca de los propósitos del mentorazgo. Ella decía que:

De la misma manera que la investigación sobre las prácticas de enseñanza ha encontrado quelos profesores y las escuelas ejercen una influencia conservadora en los alumnos en prácticas, algunos estudios muestran que los mentores promueven normas y prácticas convencionales que limitan los objetivos de la reforma. Estos resultados no deberían de sorprendernos. Los profesores mentores tienen poca experiencia con las actividades propias del mentorazgo: observar y discutir con colegas. La mayoría de los profesores trabajan solos en la privacidad de su aula, protegidos por las normas de autonomía y de no interferencia. Ni la cultura de la enseñanza anima la distinción entre profesores basada en el saber hacer. La persistencia de la privacidad, la falta de oportunidades de observar y de discutir sobre la propia práctica y la de otros y la tendencia a tratar a todos los profesores por igual limita lo que los mentores pueden hacer. Además, pocos mentores practican el tipo de enseñanza centrada en el alumno que se plantea desde las reformas actuales. Si queremos que los mentores ayuden a los profesores principiantes a aprender las formas de pensar y actuar asociadas a los nuevos modelos de enseñanza, debemos de situarlos con profesores mentores que ya han participado en la reforma de su escuela y de su enseñanza. (FEIMANNEMSER, 2001, p.2).

Por lo anterior, hemos de entender que los programas de inserción han de incluir más experiencias formativas que exclusivamente la figura del mentor. Experiencias centradas en la práctica, en las necesidades de los alumnos, en la participación en proyectos de innovación, en el contacto e intercambio con otros profesores principiantes, etc. pueden ayudar a los profesores a comprender la complejidad del acto de enseñar. Y a ello puede contribuir el desarrollo de estándares de calidad para los profesores principiantes. Decía Darling-Hammond que

la palanca política más importante de que se dispone para mejorar el proceso de enseñanza y aprendizaje es, probablemente, el desarrollo de unos estándares profesionales que contemplen los aspectos más importantes de la profesión docente. (DARLING-HAMMOND, 2001, p. 391).

Los estándares pueden ayudar también a romper ese círculo de socialización al que anteriormente nos referíamos, al contemplar objetivos de enseñanza, de actuación y competencia docente más amplios y ambiciosos.

Uno de los problemas que tiene el periodo de inserción es el de establecer los vínculos posibles entre la formación inicial (normalmente a cargo de instituciones universitarias) y la formación continua del profesorado. Como hemos comentado anteriormente, las investigaciones muestran que la formación inicial parece perder su influencia una vez que el profesorado accede como profesor permanente en un aula. Es la "laguna de los dos mundos" a la que se referían Feiman y Buchman, (2001) hace ya algunos años. Para unir estos dos mundos nos ha parecido de interés la experiencia inglesa en relación al documento denominado CEDP (Career Entry Development Profile), que es un documento que se elabora al final del periodo de formación inicial indicando los aspectos generales de la formación recibida por la persona, la edad de los alumnos que puede enseñar, y que además 
incluye una lista de áreas destacadas del profesor en relación con los estándares y cuatro áreas que se podrían mejorar a través del primer año de enseñanza (PARKINSON; PRITCHARD, 2005). Experiencias de este tipo pueden ayudar a ver las posibilidades de interrelación entre las dos instancias formativas más importantes para el profesor principiante.

Pero si algo hemos de destacar como importante para el éxito de cualquier programa de inserción es el compromiso de toda la escuela, incluyendo por supuesto a su equipo directivo, en apoyar e integrar a los nuevos profesores. Y aquí es donde vemos las mayores dificultades. A menos que se mantenga una línea de acción política de apoyo y recursos continuado al desarrollo profesional docente centrado en la escuela, que permita que el programa de inserción forme parte del proyecto educativo y de mejora de la escuela, el profesorado principiante seguirá siendo una isla, un extraño que debe de aprender a resolver sus problemas por sí solo porque así se ha hecho siempre en la escuela.

\section{Referências}

ARENDS, R.; RIGAZIO-DIGILIO, A. Beginning teacher induction: research and examples of contemporary Practice. ERIC ED 450074, 2000.

BERLINER, D. C. A personal response to those who bash teacher education. Journal of Teacher Education, v. 51, n.5, p. 358371, 2000.

BRANSFORD,J.;DARLING-HAMMOND, L.; LEPAGE, P. Introduction. In: DARLINGHAMMOND, L.; BRANSFORD, J. (Eds.). Preparing teachers for a changing world . S. Francisco: Jossey Bass, 2005, p. 1-39.
BRITTON, E. et al. Comprehensive teacher induction. Dordrecht: Kluwer Academic Press, 2003.

BUCHBERGER, F. et al. Teacher education in Europe: diversity versus uniformity. In: GALTON, B. (Ed.). Handbook of teacher training in Europe. London: David Fulton, 1997, p. 14-50.

COCHRAN-SMITH, M.; FRIES, M. The AERA panel on research and teacher education: context and goals. In:

ZEICHNER, K. (Eds.), Studying teacher education: the report of the AERA panel on research and teacher education. Mahwah, NJ: Erlbaum, 2005.

COLLIS, B.; WINNIPS, K. Two scenarios for productive learning environments in the workplace. British Journal of Educational Technology, v. 33, n.2, p. 133-148, 2002.

DARLING-HAMMOND, L. El derecho de aprender: crear buenas escuelas para todos. Barcelona: Ariel, 2001.

FEIMAN-NEMSER, S. From preparation to practice: designing a continuum to strengthen and sustain. Teaching. Teachers College Record, v. 103, n.6, 2001, p. 10131055.

FEIMAN-NEMSER, S.; BUCHMAN, M. Lagunas de las prácticas de enseñanza de los programas de formación del profesorado. In: VILLAR ANGULO, L. M. (Ed.). Conocimiento, creencias y teorías de los profesores. Alicante: Marfil, 1998.

MARCELO, C. Estudio sobre estrategias de inserción profesional en Europa. Revista Iberoamericana de Educación, n.19, p. 101-144, 1999.

MARCELO, C. Aprender a enseñar para la sociedad del conocimiento. [Electronic Version]. Educational Policy Analysis Archives, 10, 2002. Retrieved 10-08-2006 from http://epaa.asu.edu/epaa/v10n35/. 
INGERSOLL, V.; SMITH, T. The wrong solution to the teacher shortage. Educational Leadership, v. 60 n.8, p. 30-33, 2003.

INGVARSON, L.; MEIERS, M.; BEAVIS, A. Factors affecting25 the impact of professional development programs on teachers' knowledge, practice, student outcomes \& efficacy. Educational Policy Analysis Archives, v. 13 n.10, 2005.

KENNEDY, $M$. The role of preservice teacher education. In: L. DARLINGHAMMOND; SYKES; G. (Ed.), Teaching as a learning profession. Handbook of Policy and Practice. S. Francisco: JosseyBass, 1999, p. 54-85.

LORTIE, D. School teachers: a sociological study. Chicago: University of Chicago Press, 1975.

OCDE. Teachers matter: attracting, developing and retaining effective teachers. Paris: OCDE, 2005.

PAJARES, M. F. Teachers' beliefs and educational research: cleaning up a messy construct. Review of Educational Research, v. 62, n.3, p. 307-332, 1992.

PARKINSON, J.; PRITCHARD, J. The induction experiences of new qualified secondary teachers in England and Wales. Journal of In-Service Education, v. 31 n.1, p. 63-81, 2005.

RICHARDSON, V.; PLACIER, P. Teacher change. In: RICHARDSON, V. (Ed.). Handbook of Research on Teaching. Fourth Edition, New York: American Educational Research Association, 2001, p. 905-947.

SABAR, N. From heaven to reality through crisis: novice teachers as migrants. Teaching and Teacher Education, v. 20, p. 145-161, 2004.
SERPELL, Z. Beginning teacherinduction: a review of the literature (V. ERIC ED 443783). Washington: American Association of Colleges for Teacher Education, 2000.

SMITH, T.; INGERSOLL, V. What are the effects of induction and mentoring on beginning teachers turnover? American Educational Research Journal, v. 41, n. 3, p. 681-714, 2004.

VALLI, L. Beginning teacher problems: areas for teacher education improvement. Action in Teacher Education, v. XIV, n. 1, p. 18-25, 1992.

VEENMAN, S. Perceived problems of beginning teachers. Review of Educational Research, v. 54, n. 2, p. 143-178, 1984.

VONK, J. H. C. A knowledge base for mentors of beginning teachers: result of a dutch experience. In: MCBRIDGE, R. (Ed.) Teacher Education Policy London: Falmer Press, 1996, p. 112-134.

WIDEEN, M.; MAYER-SMITH, J.; MOON, B. A critical analysis of the research on learning to teach: making the case for an ecological perspective on inquiry. Review of Educational Reseach, v. 68, p. 130-178, 1998.

WONG, H. Induction programmes that keep new teachers teaching and improving. National Association of Secondary School Principal, NASPP Bulletin, v. 88, n. 638, p. 41-59, 2004.

ZEICHNER, K. M. Teacher induction practices in US and Great Britain. Paper presented at the AERA, San Francisco, 1979.

Enviado em: 13/10/2012

Aceito em: 25/11/2012 